\title{
Retrieval of a Partially Deflated Balloon: A Novel Approach
}

\author{
Stephen Nageotte, MD, MBA*, Cheryl Takao, MD \\ Division of Cardiology, Department of Pediatrics, Children's Hospital of Los Angeles, Los Angeles, California, USA
}

\begin{abstract}
A rare complication of balloon dilation in the catheterization laboratory is the inability to deflate the balloon catheter. In the literature, methods described for deflating the balloon all involve puncture or rupture of the balloon while it is within the patient. Here we present a case in which a novel approach was used in order to puncture and deflate the balloon outside of the patient. We further looked at how balloons rupture when overinflated and the potential risks associated with doing this inside of a patient.

Copyright (c) 2018 Science International Corp.
\end{abstract}

\section{Key Words}

Catheterization - Pulmonary artery stenosis - Balloon malfunction

\section{Introduction}

The inability to deflate an angioplasty balloon is a known, albeit uncommon, complication of balloon dilation procedures. Typically, methods for balloon deflation in the literature include over-inflation of the balloon in order to rupture it, inserting the stiff end of a wire through another catheter in order to puncture the balloon or insertion of a needle through the chest wall. These procedures are potentially a high risk in that they involve either rupture of the balloon within the patient or transcutaneous needle puncture. We describe a case in which an alternative approach was used for retrieval of a partially deflated balloon.

Fax +1 2037853346

E-Mail: jshd@scienceinternational.org

http://structuralheartdisease.org/

\author{
(C) 2018 Journal of Structural Heart Disease \\ Published by Science International Corp. \\ ISN 2326-4004 \\ Accessible online at: \\ http://structuralheartdisease.org/
}

\section{Case Presentation}

A 3-year-old female with a history of tetralogy of Fallot with Pulmonary Atresia status-post full repair presented with significant continued branch Pulmonary Artery (PA) stenoses. Her past medical history included being an ex-28 week premature infant, chronic lung disease, central sleep apnea, and right femoral vein thrombosis. She had two previous cardiac surgeries (a modified Blalock-Taussig shunt (BTS) in the neonatal period; central pulmonary arterioplasty and VSD repair with $14 \mathrm{~mm}$ RV to PA conduit at 9 months of age). She also had three previous catheterizations which included angioplasty of left (L)PA and right (R) $P A$, as well as implantation of a Valeo $9 \mathrm{~mm} \times 17 \mathrm{~mm}$ stent (Bard Peripheral Vascular, Tempe, AZ) in the LPA, mounted on a $7 \mathrm{~mm}$ balloon. Despite this, she had evidence of continued branch PA stenosis with RPA peak gradient of $43 \mathrm{mmHg}$ and LPA peak gradient of $46 \mathrm{mmHg}$ by echocardiogram. She was referred to the catheterization laboratory to evaluate her branch PAs with possible angioplasty and/ or stent implantation.

In the catheterization laboratory, access was first obtained in the right internal jugular vein with a $4 \mathrm{Fr}$ sheath and the left femoral vein with a $6 \mathrm{Fr}$ sheath, as the patient had a history of right femoral vein occlusion. Initial hemodynamics showed the RV pressure to be $82 \%$ of the systemic pressure and there was significant intimal proliferation within the LPA stent down to $4.1 \mathrm{~mm}$ compared to $6.7 \mathrm{~mm}$ distally. A 0.018 " Platinum Plus Guidewire (Boston Scientific Corp., Marlbor-

* Corresponding Author:

Stephen Nageotte, MD, MBA

Division of Cardiology, Department of Pediatrics

Children's Hospital of Los Angeles

4650 W. Sunset Blvd., Mailstop \#34, Los Angeles, CA 90027, USA

Tel. +1 323361 8308; Fax: +1 323361 1513; E-Mail: steve.nageotte@gmail.com 


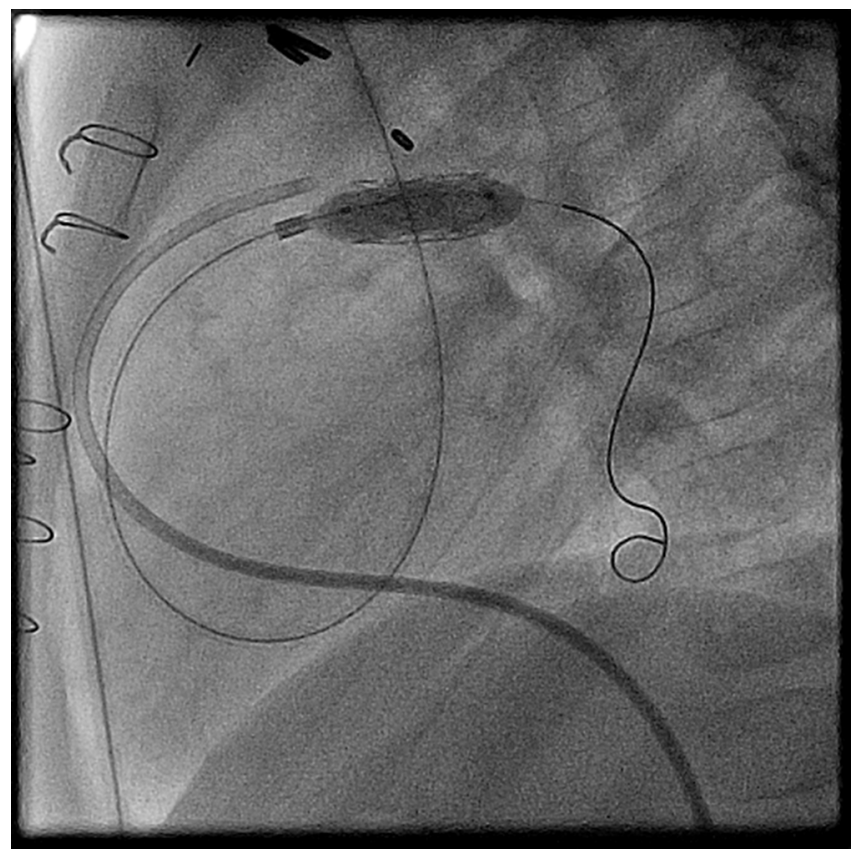

Figure 1. Partially inflated balloon within LPA stent. The $7 \mathrm{~mm} x$ $2 \mathrm{~cm}$ Sterling Balloon has been inflated within the LPA stent, but cannot be fully deflated.

ough, MA) was positioned from the RIJ sheath into the distal LPA across the LPA stent. The initial short RIJ sheath was exchanged for a $63 \mathrm{~cm}$ long $4 \mathrm{Fr}$ sheath (Cook Medical, Bloomington, IN), positioned across the LPA stent. A $7 \mathrm{~mm} \times 2 \mathrm{~cm}$ Sterling Balloon (Boston Scientific Corporation, Marlborough, MA) was advanced through the long sheath, over the wire and positioned within the LPA stent. The balloon was inflated several times until it was positioned well within the stent. After the balloon was inflated a fourth time, however, the balloon catheter could not be fully deflated and could not be housed in the $4 \mathrm{Fr}$ sheath (Figure 1). Of note, we had previously resheathed the balloon by using an inflate-deflate method involving applying positive and then negative pressure to the balloon while pulling the balloon into the sheath to keep the sheath in the pulmonary artery.

Because the balloon could not be resheathed, the sheath, balloon and Platinum Plus wire were all pulled into the right atrium to straighten the curve. Negative pressure was applied many times to the balloon with no success in deflation. The $6 \mathrm{Fr}$ sheath in

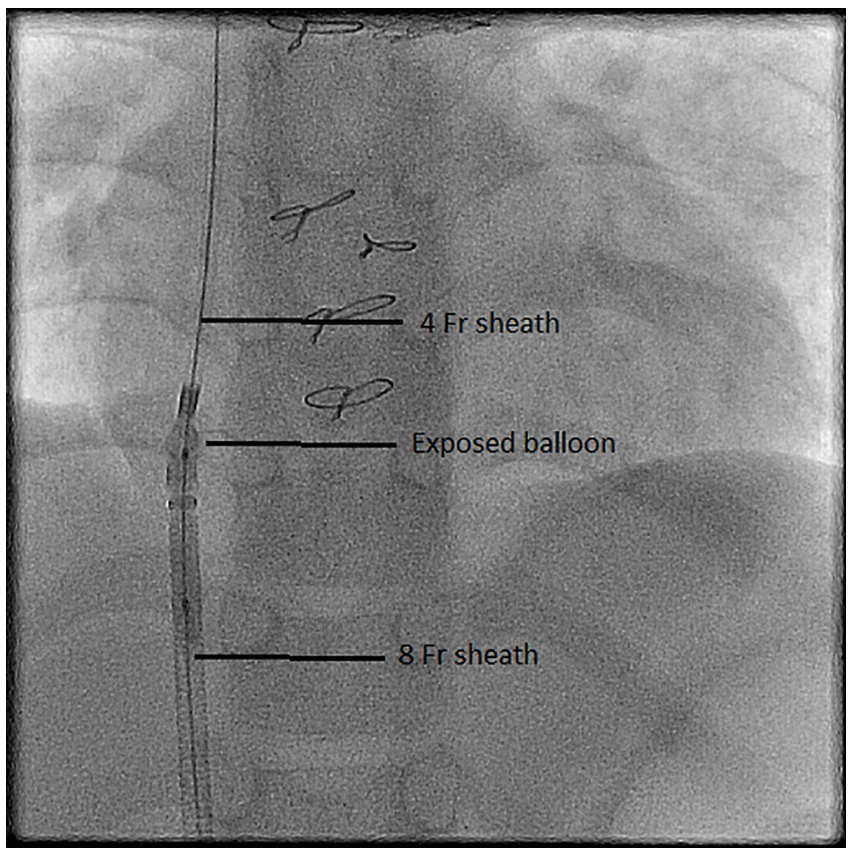

Figure 2. Balloon partially pulled into sheath. The partially deflated balloon has been snared and mostly pulled into the $8 \mathrm{Fr}$ sheath with a portion of the balloon exposed in right atrium. Note that the width of the balloon is similar to the width of the $8 \mathrm{Fr}$ sheath.

the LFV was exchanged for an $8 \mathrm{Fr} 90 \mathrm{~cm}$ long sheath in an attempt to snare and cover the deflated balloon. The distal tip of the Platinum Plus wire was snared in the right atrium through this $8 \mathrm{Fr}$ sheath and the balloon was pulled into this larger sheath. Unfortunately, the balloon could not be completely pulled into the $8 \mathrm{Fr}$ sheath, but the profile of the balloon extruding from the sheath was nearly the same width as the 8 Fr sheath (Figure 2). The $8 \mathrm{Fr}$ sheath, snare, balloon, Platinum Plus wire and $4 \mathrm{Fr}$ sheath were then pulled down and out of the left groin with part of the balloon uncovered by the sheath (Figure 3). The partially inflated balloon was exposed and punctured with a needle and deflated manually. It could then be pulled back into the $4 \mathrm{Fr}$ RIJ long sheath and safely removed (Figure 4).

\section{Discussion}

The inability to deflate an angioplasty balloon is a known, albeit uncommon, complication of balloon dilation procedures [1]. This complication was first 


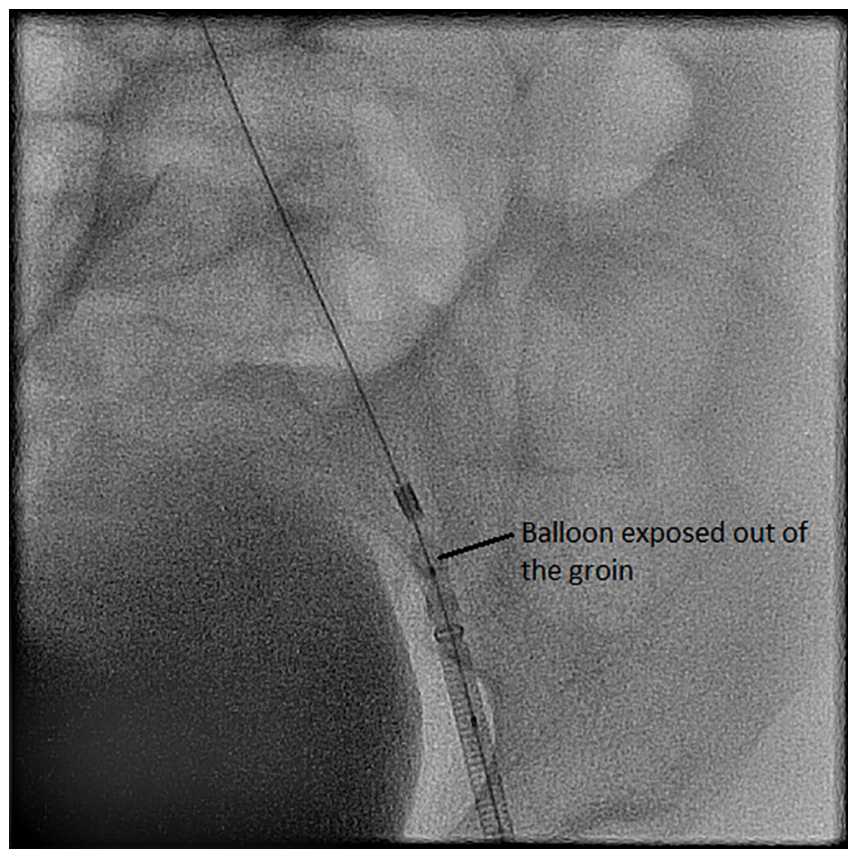

Figure 3. Balloon pulled out of the groin. The balloon, mostly within the $8 \mathrm{Fr}$ sheath, has been pulled out of the left groin so that it could be exposed and popped manually.

described in the early literature for the balloon atrial septostomy procedure [2-6]. In some cases, successful deflation of the balloon was eventually achieved by use of the stiff end of a wire advanced either through the second lumen of the balloon catheter or through a second end-hole catheter. With the wire held in position just past the tip of the catheter, the balloon was pulled back onto the wire in order to puncture the balloon $[2,5]$. In other cases, the balloon was punctured by a fine needle introduced percutaneously, either transhepatically or through the chest wall $[3,4]$. Both of these approaches have significant risks associated with them. With the first approach, there is the risk of vessel/cardiac damage from the wire. With the latter, there are obvious risks through transcutaneous needle access of the balloon of damage to the heart and surrounding structures. According to Hijazi et al., when a balloon septostomy catheter does not deflate, the first thing to do is to pass a guide wire in the balloon lumen to clear any obstruction. This maneuver was not performed in our case. If this does not work, an injector should be connected to the balloon and 3-5 cc of contrast injected under pressure using 300 psi in order to rupture the balloon [7]. With this

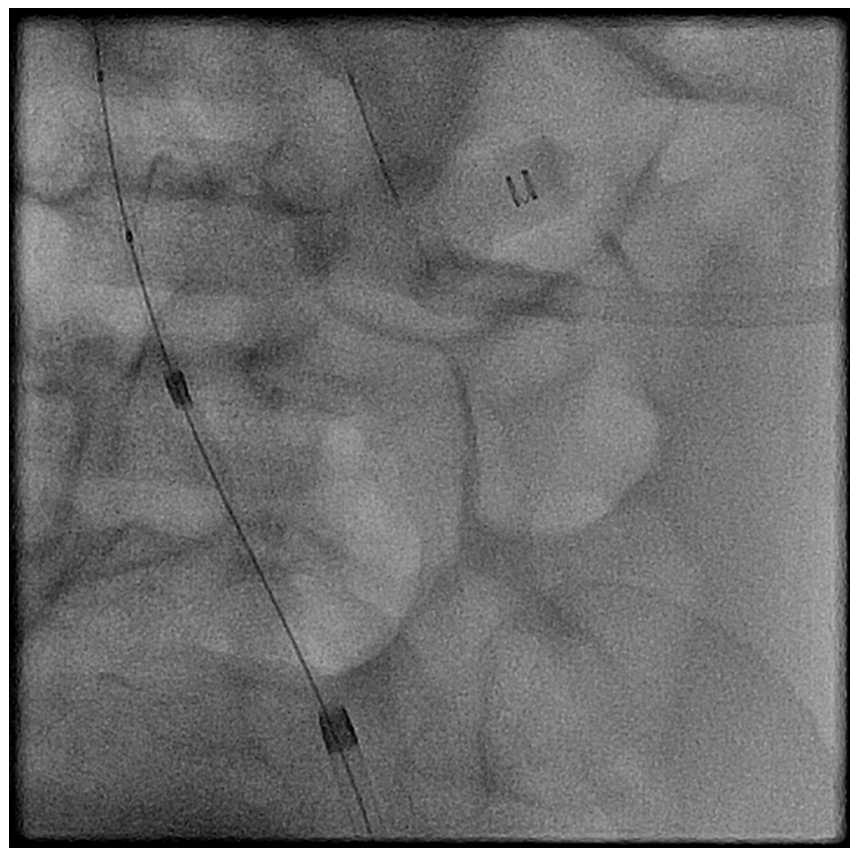

Figure 4. Deflated balloon within sheath. After the balloon has been manually deflated, it has now been pulled back into 4 Fr RIJ sheath so that it can safely be removed from the body.

approach, rupture of the balloon at high pressure has the risk of embolization of the balloon fragments[8]. Finally, if this does not work, one can try using the stiff end of a guide wire through a second catheter [7].

In order to test the technique of balloon rupture, we overinflated a series of balloon catheters ex vivo to see at what pressure the balloons burst and how they tore. First, we manually burst a series of balloons by slowly over-inflating the balloon with a BasixTouch inflation device (Merit Medical, South Jordan, UT) until the balloon burst. It should be noted that balloon rupture mechanisms are subject to variations due to balloon materials system fatigue. Table 1 lists the sizes and types of balloons tested as well as the burst pressures and type of hole or tear created. During manual over-inflation, all Sterling balloons as well as the Opta Pro, Palmaz Blue and Valeo balloons had longitudinal tears. The Dorado Balloon burst with a pinhole tear in the proximal balloon. The Atlas Gold Balloon did not burst, but the high pressure created a connection between the wire lumen and the balloon lumen within the catheter. This balloon remained unable to deflate. We additionally burst a Miller-Edwards Balloon Septostomy Catheter (Edwards Life sciences, Irvine, CA). 
Table 1. Manual burst pressures.

\begin{tabular}{|c|c|c|c|c|c|}
\hline \multicolumn{6}{|c|}{ Manual Burst } \\
\hline Size $(\mathrm{mm} \times \mathrm{cm})$ & Type & Burst (atm) & Burst (psi) & Listed Burst (atm) & Type of hole/ tear \\
\hline $5 \times 2$ & Sterling & 26 & 382 & 14 & Longitudinal \\
\hline $5 \times 2$ & Sterling & 27 & 397 & 14 & Longitudinal \\
\hline $6 \times 2$ & Sterling & 24 & 353 & 14 & Longitudinal \\
\hline $7 \times 2$ & Sterling & 27 & 397 & 14 & Longitudinal \\
\hline $16 \times 2$ & Atlas Gold & 35 & 514 & 18 & $\begin{array}{l}\text { Hole between wire } \\
\text { and balloon lumens }\end{array}$ \\
\hline $8 \times 2$ & Opta Pro & 17 & 250 & 10 & Longitudinal \\
\hline $7 \times 2$ & Dorado & 27 & 397 & 22 & Proximal Pinhole \\
\hline $6 \times 1.7$ & Palmaz Blue & 22 & 323 & 10 & Longitudinal \\
\hline $7 \times 1.8$ & Valeo & 27 & 397 & 14 & Longitudinal \\
\hline \multicolumn{2}{|c|}{ Miller-Edwards BAS balloon } & 6 & 88 & & $\begin{array}{l}\text { Complete rupture of } \\
\text { balloon }\end{array}$ \\
\hline
\end{tabular}

Atm= atmospheres; $p s i=$ pounds per square inch

Table 2. Power burst pressures.

\section{Power Burst}

\begin{tabular}{llllll}
\hline Size $(\mathbf{m m} \mathbf{~ x m})$ & Type & Burst (atm) & Burst (psi) & Listed Burst (atm) & Type of hole/ tear \\
\hline $5 \times 2$ & Sterling & 27 & 401 & 14 & Longitudinal \\
$6 \times 2$ & Sterling & 33 & 481 & 14 & Longitudinal \\
$8 \times 2$ & Sterling & & & 12 & Hole between wire \\
and balloon lumens
\end{tabular}

At $6 \mathrm{~mL}$ of fluid and at a pressure of 6 atm, the balloon burst and the fragment completely ruptured off of the catheter (Figure 5)(Table 1).
Next, we tested the balloon bursts in the manner Hijazi et al. proposed using a pressure injection. When we did this in the manner described, the power 


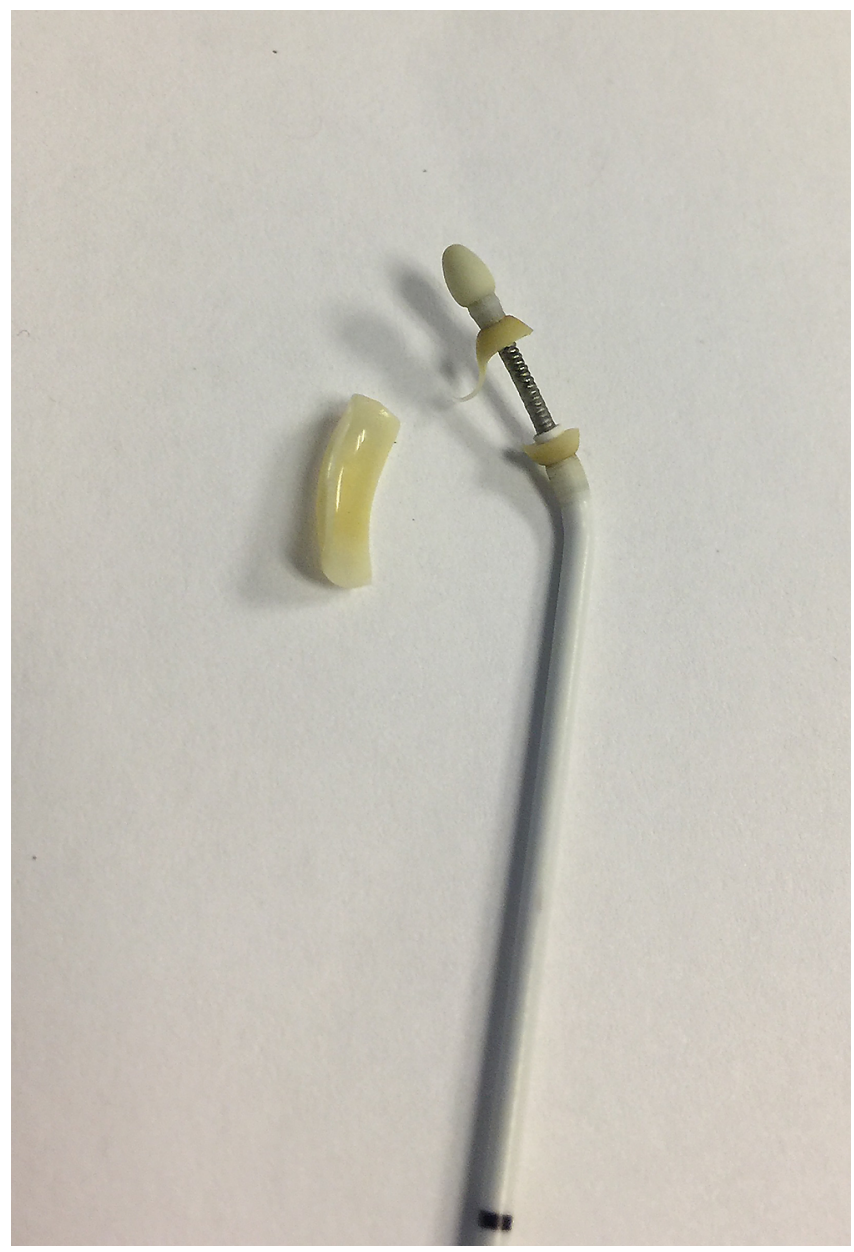

Figure 5. Complete rupture of BAS balloon. Shown is a ruptured Miller-Edwards Balloon Septostomy Catheter with balloon fragment seen.

kept auto-stopping due to the peak pressure limit of 300 psi. When we changed the peak pressure limit to 600 psi, we were able to burst the balloons. Table 2 similarly lists the sizes and types of balloons tested as well as the burst pressures and type of hole or tear created, again keeping in mind that balloon rupture mechanisms are subject to variations due to balloon materials system fatigue. Unfortunately, not all burst pressures were captured. Both Dorado balloons developed pinhole tears in the proximal balloon. Additionally, the Atlas Gold as well, as one Sterling balloon, developed a hole between the wire and balloon lumens. However, these balloons did eventually deflate (Table 2).
In our patient, by covering the balloon with the larger sheath, the balloon was able to be positioned outside of the body where it could be punctured. Multiple factors contributed to the success of this technique and may not be applicable in many other situations. In our case, the balloon was not stuck fully inflated to its maximal diameter and thus could be safely pulled back into the right atrium. If there was a structure proximal to the balloon that was narrower than the partially inflated balloon, it could not have been pulled safely into the right atrium, making our technique more difficult. In the selection of the size of the second venous sheath, careful attention needed to be paid to the size of the partially deflated balloon. While our intent was to fully pull the partially inflated balloon into the larger sheath, luckily the exposed balloon was nearly the exact size of the sheath, making it safe to remove from the vessel orifice partially exposed. An even larger sheath could have been used to fully cover the balloon. This technique could not be performed if the angioplasty was being performed on the arterial side. Finally, if the balloon did not partially deflate, it could not have been pulled out of the stent and would have obstructed flow to the LPA. In this scenario, one of the other techniques to rupture the balloon in its position could be employed.

\section{Conclusion}

We report a novel approach to removal of a balloon that could not be fully deflated. Utilizing this method does not involve puncture or rupture of the balloon while it is still inside the patient. A second venous access point opposite to the site where the balloon catheter enters often can straighten the balloon catheter and assist in its retrieval - the largest size sheath that can be placed safely should be considered to fully cover the balloon. While there are limitations to this approach, removal of a partially deflated balloon in this manner offers a safe alternative to the traditional removal techniques. As new balloon catheters emerge, it is important to know how they will rupture if one plans to rupture the balloon within the patient as there may be a risk of balloon embolization, pinhole balloon rupture or creation of a connection between the wire and balloon lumens. If the patient is stable, it may be worthwhile to first attempt balloon 
rupture outside of the patient with the same type of balloon. However, it should be noted that in vivo rupture mechanics of the balloon can differ when they are subjected to the stresses of use during catheterization. Perhaps more important than the specifics of this technique, is the notion that when faced with difficult clinical situations, the interventional cardiologist needs to think outside the box.

\section{Conflict of Interest}

The authors have no conflict of interest relevant to this publication.

\section{Comment on this Article or Ask a Question}

\section{References}

1. Mullins CE. Cardiac Catheterization in Congenital Heart Disease: Pediatric and Adult. Wiley-Blackwell, 2006:391,428. DOI: 10.1002/9780470986967

2. Ellison RC, Plauth $W H$, Gazzaniga $A B$, Fyler $D C$. Inability to deflate catheter balloon: a complication of balloon atrial septostomy. J. Pediatr. 1970:76;604-606. DOI: 10.1016/ S0022-3476(70)80412-7

3. Wilson NJ, Culham JA, Sandor GG. Successful treatment of a nondeflatable balIoon atrial septostomy catheter. Pediatr. Cardiol. 1990:11;150-152. DOI: 10.1007/ BF02238846

4. Scott O. A new complication of Rashkind balloon septostomy. Arch. Dis.
Child.1970:45;716-717. DOI: 10.1136/ adc. 45.243 .716

5. Ozkutlu S, Ozbarlas N. Successful treatment of a nondeflatable balloon atrial septostomy catheter. Int. J. Cardiol. 1992:34;348350. DOI: 10.1016/0167-5273(92)90036-3

6. Hohn AR, Webb HM. Balloon deflation failure: a hazard of 'medical' atrial septostomy. Am. Heart J. 1972:83;389-391. DOI: 10.1016/0002-8703(72)90441-3

7. Hijazi ZM, Feldman T, Cheatham JP, Sievert H. Complications During Percutaneous Interventions for Congenital and Structural Heart Disease. UK, Informa Healthcare Ltd; Fl, Taylor \& Francis Group, 2009:48. DOI: $10.3109 / 9780203092118$
8. Vogel JH. Balloon embolization during atrial septostomy. Circulation 1970:42;155156. DOI: 10.1161/01.CIR.42.1.155

Cite this article as: Nageotte $\mathrm{S}$, Takao $\mathrm{C}$. Retrieval of a Partially Deflated Balloon: A Novel Approach. Structural Heart Disease. 2018;4(5):228-233. DOI: https:// doi.org/10.12945/j.jshd.2018.041.17 